\title{
Image Clustering Method Based on Density Maps Derived from Self-Organizing Mapping: SOM
}

\author{
Kohei Arai \\ Graduate School of Science and Engineering \\ Saga University \\ Saga City, Japan
}

\begin{abstract}
A new method for image clustering with density maps derived from Self-Organizing Maps (SOM) is proposed together with a clarification of learning processes during a construction of clusters. It is found that the proposed SOM based image clustering method shows much better clustered result for both simulation and real satellite imagery data. It is also found that the separability among clusters of the proposed method is $16 \%$ longer than the existing k-mean clustering. It is also found that the separability among clusters of the proposed method is $16 \%$ longer than the existing k-mean clustering. In accordance with the experimental results with Landsat-5 TM image, it takes more than 20000 of iteration for convergence of the SOM learning processes.
\end{abstract}

Keywords- Clustering; self organizing map; separability; Learning process; Density map; Pixel labeling; Un-supervised classification.

\section{INTRODUCTION}

Clustering method is widely used for data analysis and pattern recognition [1]-[4]. Meanwhile, Self-Organizing Map: SOM proposed by $\mathrm{T}$. Kohonen is a neural network with two layers which allows use as un-supervised classification, or learning method [5] based on a similarity between separable data groups to be classified [6]. In other word, SOM is a visualization tool for multi-dimensional data rearranging the data in accordance with a similarity based on a learning process with the statistical characteristics of the data. It is used to be used for pattern recognition in combination with Learning Vector Quantization $\left(\mathrm{LVQ}^{1}\right)$. SOM is consists of $\mathrm{m}$ dimensional input layer which represent as a vector and two dimensional output layer which is also represented as a vector connected each other nodes between input and output layers with weighting coefficients. In a learning process, winning unit is chosen based on the difference between input vector and weighting coefficients vector then the selected unit and surrounding units get closer to the input vector.

SOM is utilized for clustering [7]. After a learning process, a density map ${ }^{2}$ is created in accordance with code vector

http://en.wikipedia.org/wiki/Learning_Vector_Quantization

http://books.google.co.jp/books?id=wxvQoFy1 YBgC\&pg=SA 1-PA210\&lpg=SA1-

PA210\&dq=density + map+SOM\&source=bl\&ots=sU95Gi28u g\&sig=uZBXSATAqYaXPJtkmrGHts7uoqU\&hl=ja\&sa=X\&e density. Based on the density map, a pixel labeling ${ }^{3}$ can be done. This is the basic idea on the proposed image clustering method with SOM learning. Other than this, clustering methods with learning processes, reinforcement learning is also proposed for image retrievals [8] and rescue simulations [9]. Also probability density model for SOM is proposed.

The image clustering method with SOM learning based on density map is proposed in the following section followed by experimental results with satellite remote sensing imagery data. Then finally, conclusions and some discussions are described.

\section{Proposed IMAGE Clustering Method}

Firstly imagery data are mapped to a feature space. In parallel, SOM learning process creates a density map in accordance with a similarity between the mapped data in the feature space and density map or between input data in the feature space and two dimensional density maps. As a result of SOM learning process, code vector is obtained. It is easy to recognize the density of the code vector visually. Although code vector density map represent cluster boundaries, it is not easy that neither to determine a boundary nor to put a label to the pixel in concern by using the density map. The method proposed here is to use density map for finding boundaries among sub-clusters then some of sub-clusters which have a high similarity are to be merged in the following procedure,

(1)Create density map based on SOM learning

(2)Binary image is generated from the density map

(3)Define sub-clusters in accordance with the separated areas of the binary image

(4)Calculate similarities of the sub-clusters

(5)Merge the sub-clusters which show the highest similarity

(6)Process (4) and (5) until the number of clusters reaches the desired number of clusters

$\mathrm{i}=$ hijYT7L0CIibiQfn0NSTAw\&ved=0CGkQ6AEwBA\#v=one page $\& q=$ density $\% 20 \mathrm{map} \% 20 \mathrm{SOM} \& \mathrm{f}=$ false

http://books.google.co.jp/books?id=jJad0gh8YwC\&pg=PA69\&dq=pixel+labeling\&hl=ja\&sa=X\&ei= ZinYT4CpFYjUmAWW1cCfAw\&ved=0CDUQ6AEwAA\#v= onepage \&q=pixe1\%20labeling \&f=false 
Representing input vector, $x(t)$ and reference (or output) vector, $\mathrm{m}(\mathrm{t})$, neural network proposed by $\mathrm{T}$. Kohonen is expressed as follows,

$$
m(t+1)=m(t)+h_{\mathrm{i}}(t)[x(t)-m(t)]
$$

where $h(t)$ denotes neighboring function or weighting function including learning coefficients.

$$
\begin{aligned}
h_{i}(t) & =a(t), \text { when } i \in N(t) \\
& =0, \quad \text { when } i \notin N(t)
\end{aligned}
$$

where $N(t)$ denotes the number or size of neighboring units. $a(t)$ is called learning coefficient and ranges from 0 to 1 as is expressed as follows,

$$
\mathrm{a}(\mathrm{t})=\mathrm{a}_{0}(1-\mathrm{t} / \mathrm{T})
$$

where $a_{0}$ is an initial value and $T$ denotes the number of total learning number or the number of update. In the equation (1), $[x(t)-m(t)]$ implies cost function ${ }^{4}$ which should be minimized, and if

$c=\underset{i}{\operatorname{argmin}} .\left\|x-m_{\mathrm{i}}\right\|$

is obtained then such $\mathrm{m}_{\mathrm{i}}$ unit is called winning unit. The neighboring unit is defined around $\mathrm{m}_{\mathrm{i}}$ unit. The size of the neighboring unit, $N(t)$ is a variable which starts with a relatively large then is getting small reaching to the wining unit only after the SOM learning process.

$$
N(t)=N(0)(1-t / T)
$$

The SOM learning process is illustrated in Fig.1.
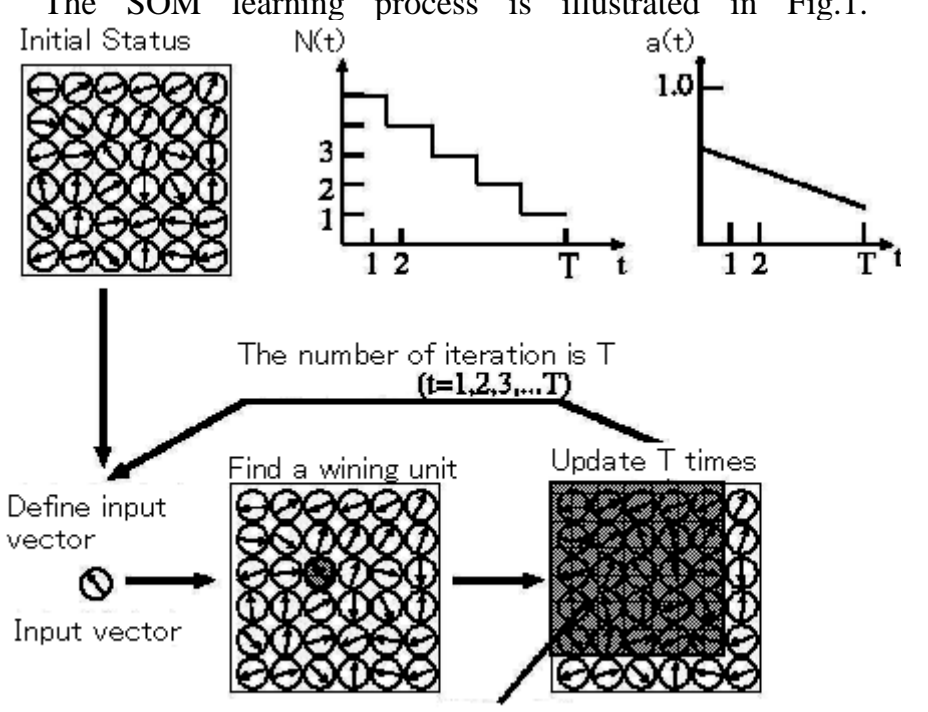

The neighboring region

Figure 1. Illustrative view of the SOM learning process

http://books.google.co.jp/books?id=AuY1PwAACAAJ \&dq=c ost+function \&hl=ja\&sa=X\&ei=6ynYT6DxA8rxmAXQlsGN Aw\&ved=0CDUQ6AEwAA
The existing clustering algorithm such as k-means clustering algorithm ${ }^{5}$ is similar to the SOM learning process. If $\mathrm{m}_{\mathrm{i}}$ is redefined as mean vector of cluster $i$, then the cost function defined in the k-means clustering is expressed as follows,

$$
J=\sum_{(6)}\left\|x(t)-m_{\mathrm{i}}(x(t))\right\|^{2}
$$

Therefore, the mean vector of each cluster is determined to minimize the equation (6) of cost function. Let $I(x(t))$ be a binary function and is equal to 1 if the $x(t)$ belongs to the cluster $\mathrm{i}$ and is 0 if the $x(t)$ does not belong to the cluster $i$, then the cost function can be rewritten as follows,

$$
J^{\prime}=\sum \sum I(x(t))\left\|x(t)-m_{i}(x(t))\right\|^{2}
$$

Meanwhile $m_{\mathrm{i}}(x(t))$ is updated as follows,

$$
m_{i}(x(t+1))=m_{i}(x(t))+\lambda I(x(t))\left\|x(t)-m_{\mathrm{i}}(x(t))\right\|
$$

It is because of the following equation.

$$
\partial J / \partial m_{\mathrm{i}}(x(t))=-2 \sum I(x(t)) \| \mathrm{x}(t)-m_{\mathrm{i}}(x(t)) \mid
$$

The k-means clustering algorithm can be rewritten as follows,

Set initial status of mean vectors of $k$ clusters, $m_{\mathrm{i}}(x(0))$, $i=1,2, \ldots, k$, then

(2)Iteration of the following two steps for $t=k+1, k+2, \ldots, N$,

$$
\begin{aligned}
& I_{i}(x(t))=1, \text { when }\left\|x(t)-m_{\mathrm{i}}(x(t))\right\| \leq\left\|x(t)-m_{\mathrm{j}}(x(t))\right\| \forall j \\
& \quad=0, \text { elsewhere }
\end{aligned}
$$

$$
m_{\mathrm{i}}(x(t+1))=m_{i}(x(t))+I(x(t)) \| x(t)-m_{i}(x(t)) \mid / \sum_{t^{\prime}=1}^{t} I\left(x\left(t^{\prime}\right)\right)
$$

The equation (11) is identical to the equation (8) if $\lambda$ is replaced to $1 / \sum I\left(x\left(t^{\prime}\right)\right)$.

The difference of input data is enhanced in the output layer unit through SOM learning so that similar code vector of the unit becomes formed. Meanwhile, if the similar input data are separated in their location each other, it becomes neighboring units in the output layer unit. Density map $f(j, k)$ is defined as follows,

$$
\begin{aligned}
& \mathrm{f}(\mathrm{j}, \mathrm{k})=\sum\left(\mathrm{m}_{\mathrm{j}, \mathrm{k}}-\mathrm{m}_{\mathrm{j}-1, \mathrm{k}-\mathrm{n}}\right)^{\mathrm{T}}\left(\mathrm{m}_{\mathrm{j}, \mathrm{k}}-\mathrm{m}_{\mathrm{j}-\mathrm{l}, \mathrm{k}-\mathrm{n}}\right) / \mathrm{D} \\
& (\mathrm{l}, \mathrm{n}) \in \mathrm{D}
\end{aligned}
$$

where $D$ is neighboring unit, 8 neighbor unit centered the unit in concern in this paper. This density map has the relation among the input imagery data, feature space and SOM learning process as is illustrated in the Fig. 2.

This is an inverse function of the similar data concentration so that the density map obtained by a SOM learning process is quite similar to the distribution in the feature space mapped

5

http://books.google.co.jp/books?id=WonHHAAACAAJ\&dq= $\mathrm{k}-$ means+clustering \&hl=ja\&sa=X\&ei=hirYT_DvF8PJmQWX8 KGgAw\&ved=0CD4Q6AEwAQ 
from the input data. An example of density map is illustrated in the Fig.3. In the figure, dark portion means dense of code vector meanwhile light portion is sparse of code vector and becomes boundary between the different clusters.

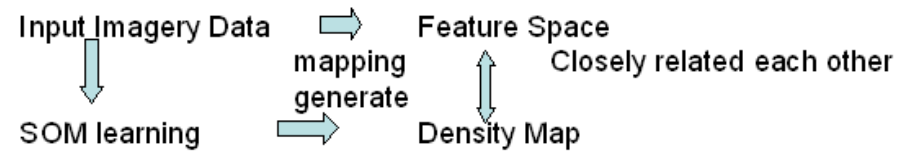

Figure 2. Relations among the input imagery data, feature space and density map generated through SOM learning.

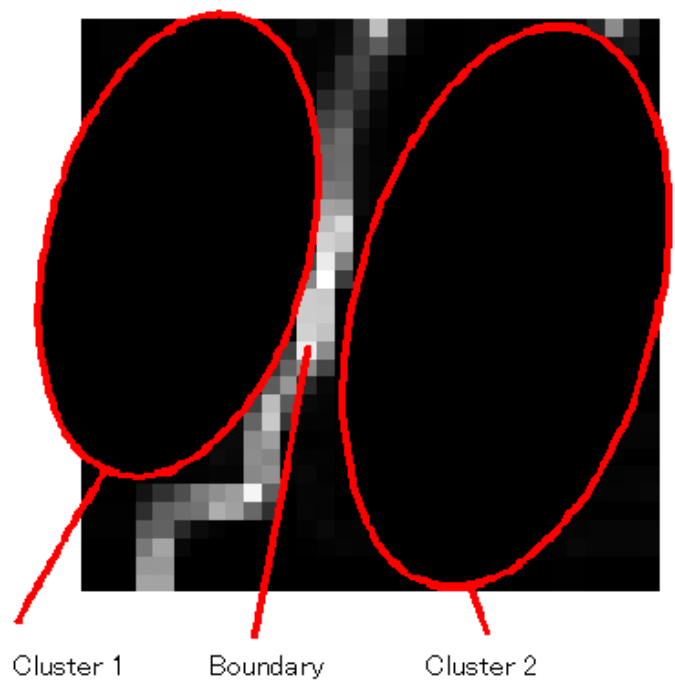

Figure 3. Example of density map as a result of SOM learning process.
1
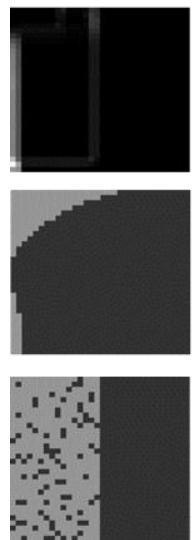

2
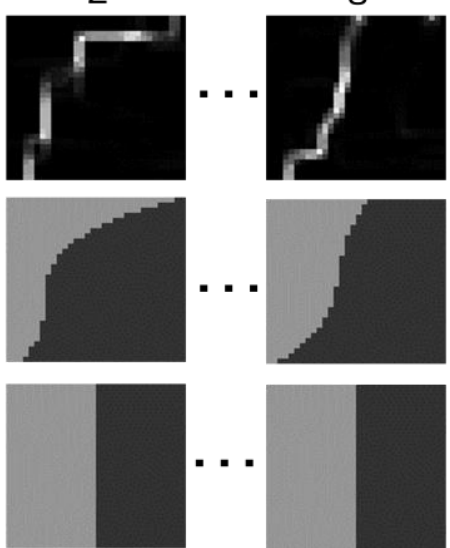

9
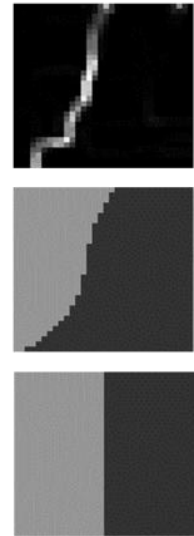

Figure 4 Example of preliminary result of density map, binarized density map and clustering result with increasing of the iteration number (multiplied by 512).

Fig.4 shows a preliminary result of density map, binarized density map and clustering result with increasing of the iteration number. In this case, initial variances of the two clusters are set at 0.03 . In accordance with the number of iteration, density map becomes clear together with binalized density map. Furthermore, cluster result becomes ideal goal.

Fig.5 shows examples density map, estimated boundary and clustered result for the easiest separate type of simulated imagery data Clustering has been done in an iterative manner. The example shows iteration number 1 to 9 as an example. Density map and estimated boundary changes by iteration by iteration results in refinement of the cluster results. Thus the proposed method may reach a final cluster result.

12
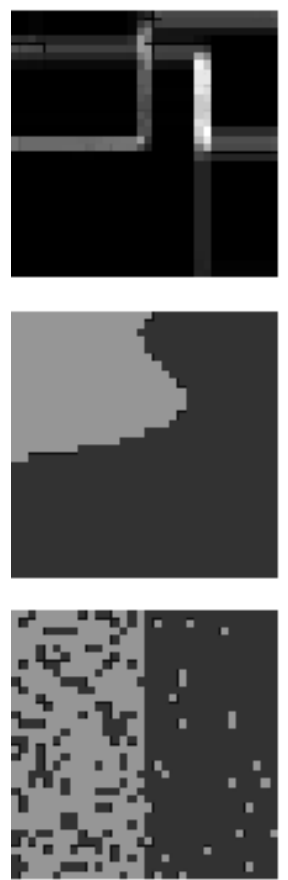
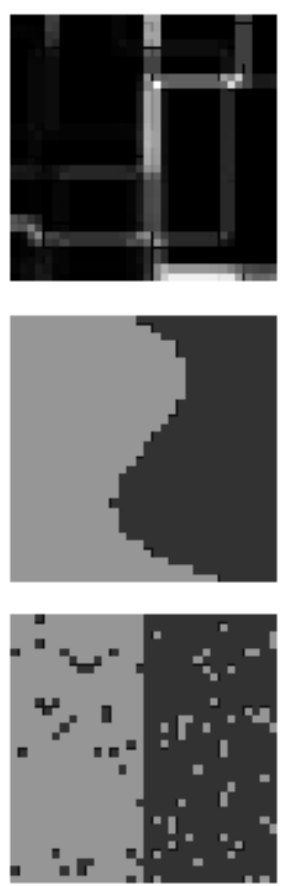

5
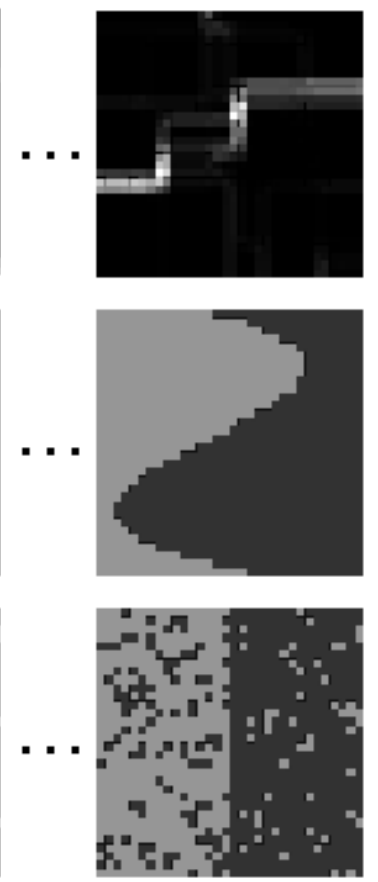

$\cdots 8$
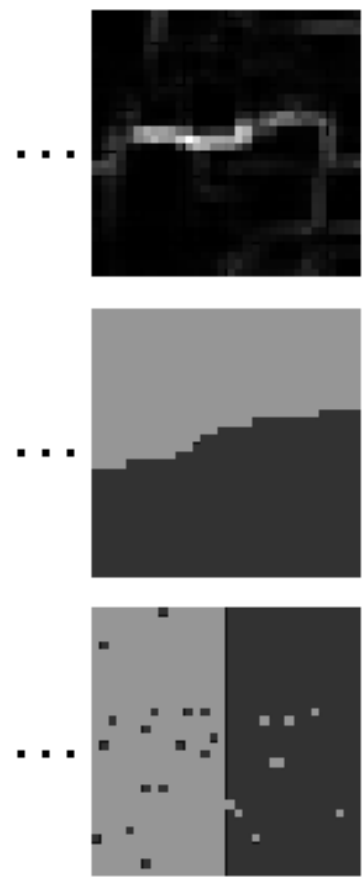

9

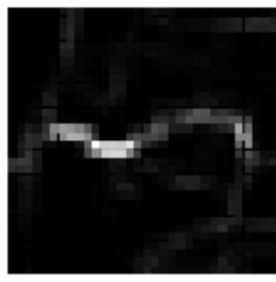

Desity

Map

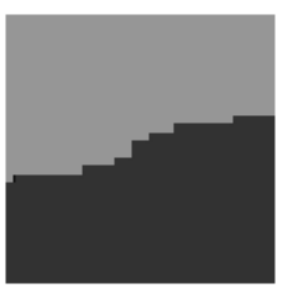

Estimated Boundary

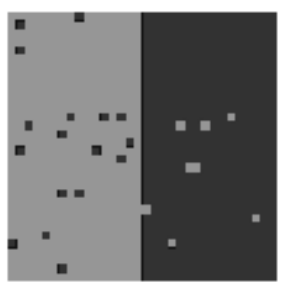

Clustered Result

Figure 5 Examples density map, estimated boundary and clustered result for the easiest separate type of simulated imagery data 


\section{EXPERIMENTS}

\section{A. Simulation Data}

Experiments with simulated imagery data and real satellite remote sensing imagery data are conducted. With a random number generator, three types of 30 sets of simulated imagery data consists of 32 by 32 pixels are generated. The first type is the most separable data set with a cluster to cluster distance, between cluster variance $\sigma_{b}=8 \sigma$ ( $\sigma$ means a within cluster variance) while the third one is the most difficult to separate data set of $\sigma_{b}=3 \sigma$ and the second one is the middle between the easiest and difficult, $\sigma_{b}=4 \sigma$. The number of clusters is set to two.

Although the original simulated images are not illustrated in the figure, it is quite obvious that the right half of image portion is cluster \#1 and the left half is cluster \#2. The top number shows the number of iteration so that SOM learning process is started from the left hand side. As is illustrated in the figure, the estimated boundary in the density map varies so remarkably. In conjunction with the changes of the density map, clustered result is varied. It is also found that the probability of the correct clustering becomes high in accordance with the number of iteration.

Also an example of SOM leaning process is shown in Fig.6. It takes a long time for the SOM learning with a relatively long between cluster distance (difficult to cluster) while it converged at the number of iteration of around 1000 for the relatively short between cluster distance (easy to cluster) as is shown in Fig.7. True simulated data consists two clusters and adjacent each other cluster at the center line of simulation data. It is shown that two clusters can be separated into two right and left regions in accordance with the iteration number, learning processes.

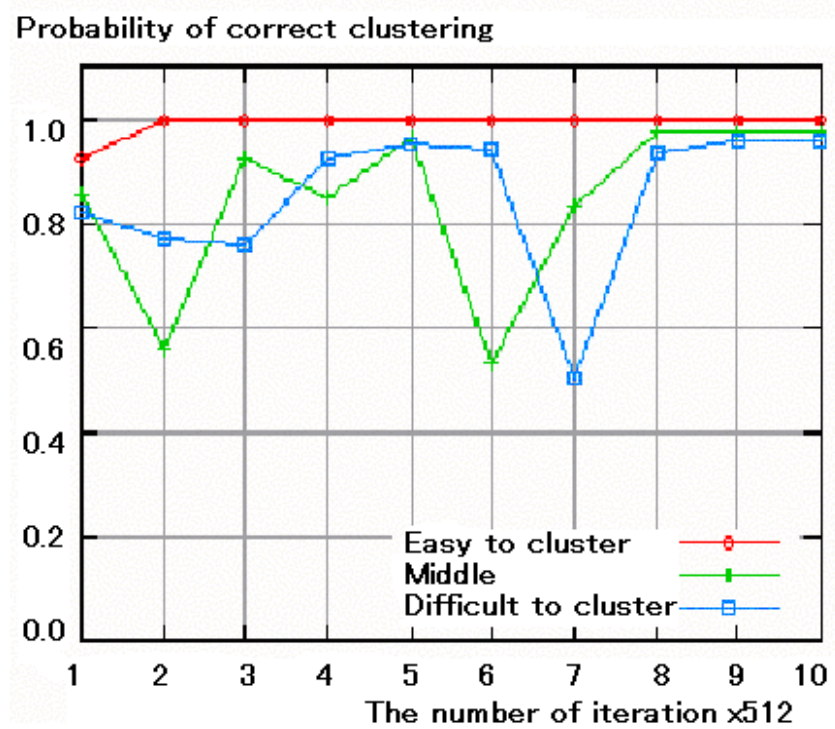

Figure 6 Example of SOM learning process for three simulation imagery data sets

\section{B. Landsat Thematic Mapper Data}

Landsat-5 TM data of Saga, Japan acquired on 15 May 1987 which is shown in Fig. 6 is used. The meta data is as follows, Entity ID: LT51130371987135HAJ00, Acquisition Date: 15-MAY-87, Path: 113, Row: 37.

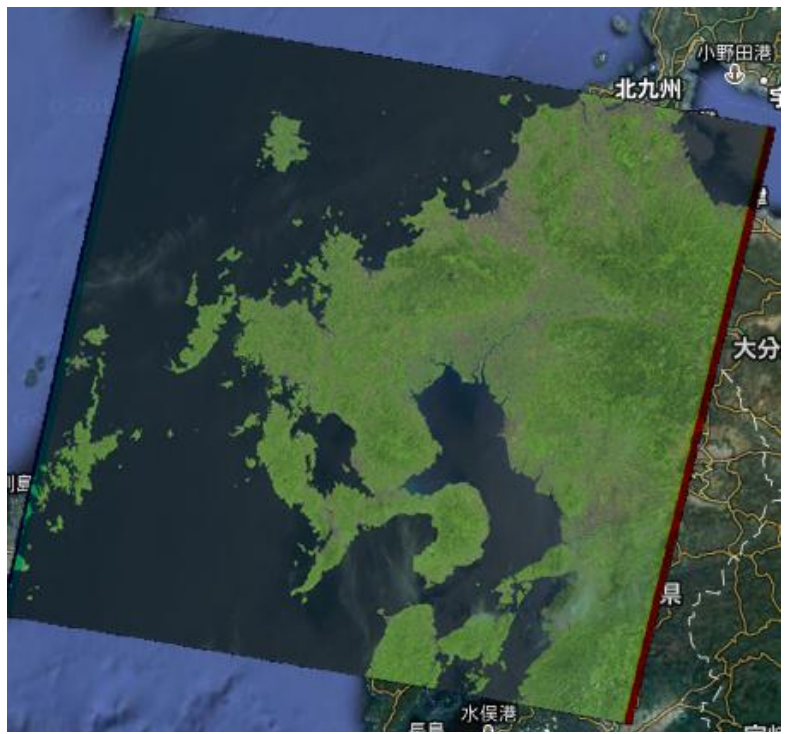

Figure 7 Landsat-5 TM image of northern Kyushu, Japan used.

Fig. 8 shows a portion of Landsat-5 TM image for each spectral band. Also, Fig.9 shows the clustered results for the proposed SOM based clustering with density map, k-mean clustering and supervised classification of Maximum Likelihood classification: MLH as well as a portion of original Landsat-5 TM image which is corresponding area to the area used. For these experiments with real remote sensing satellite imagery data, five classes or clusters, Ariake sea, Road, Paddy field, Bare soil, Artificial construction (houses) are set. By referring the corresponding topographic land use map of Saga, Japan together with the original Landsat-5 TM image, it is found that the clustered result from the proposed method is more appropriate than that from k-mean clustering and MLH. In particular, detailed portion of tiny road between paddy fields are classified with the proposed method.

SOM learning process is shown in Fig.10. In accordance with increasing of iteration number, boundaries of the density map are getting much clear. Furthermore, the clustered results become a true classified map with increasing of iteration number.

Table 1 shows confusion matrix between SOM clustering and MLH classification. Percent Correct Classification: PCC is $88.8 \%$ so that classification results for both SOM clustering and MLH classification are similar except soil and water body. Spectral characteristics of these soil and water body are quite similar. Therefore, it is understandable the poor classification performance between soil and water body. 


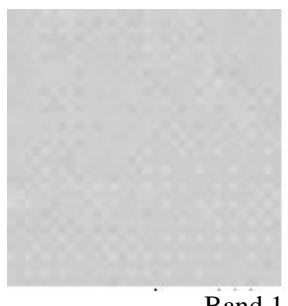

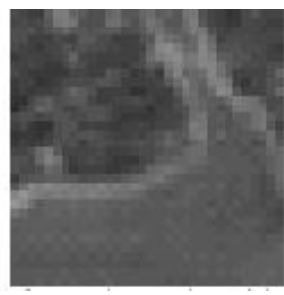

Band 2

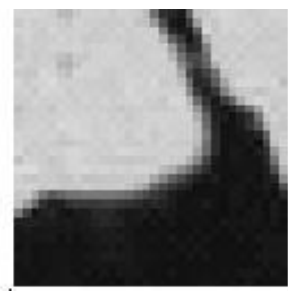

Band 4

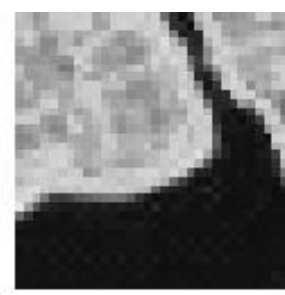

Band 5

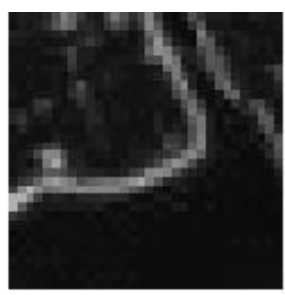

Band 7

Figure 8 Landsat-5 TM imagery data of Saga, Japan (32x32) acquired on 15 May 1987 used

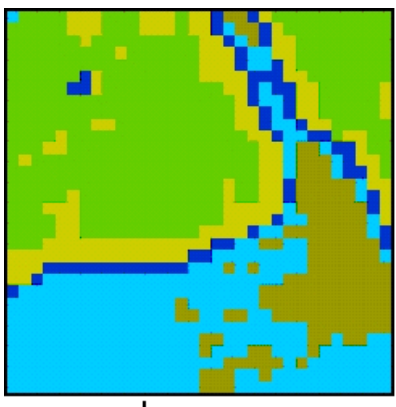

k-mean

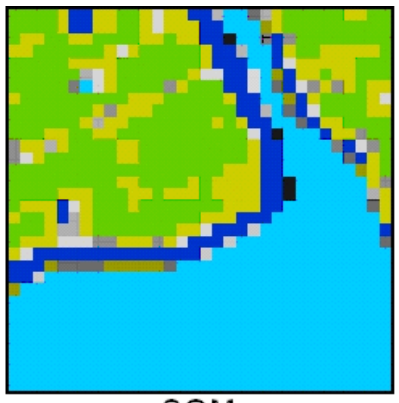

SOM

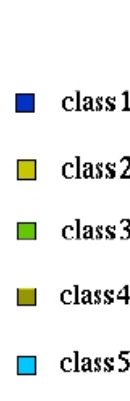

ass 5 Figure 9 Comparisons of clustered results from k-mean clustering, Maximum Likelihood classi
referring the derived density map.
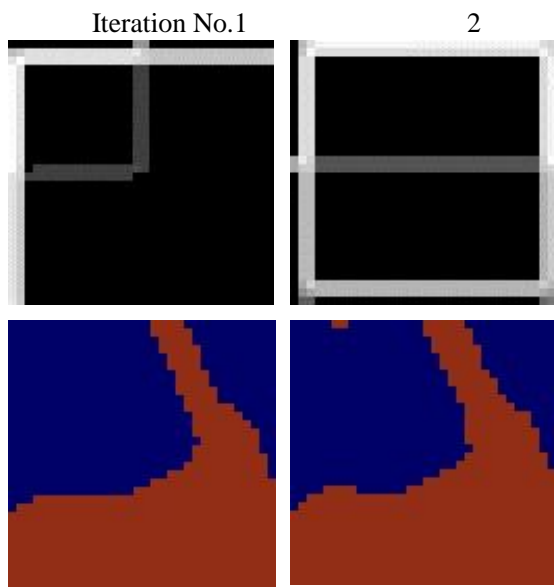

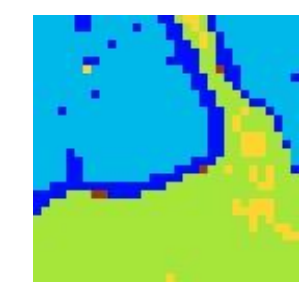

14
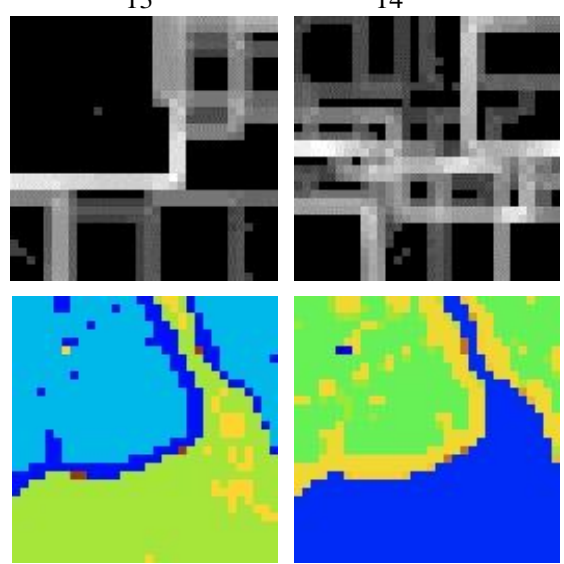

18
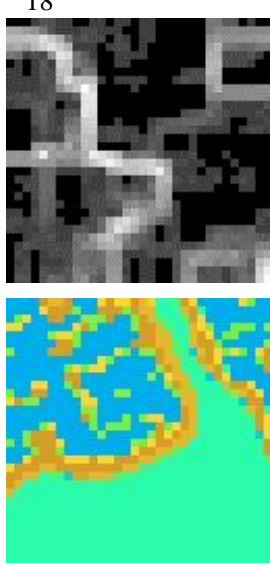

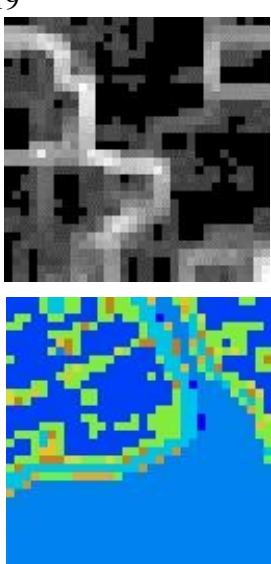

Figure 10 SOM learning process (Density map: top row, clustered result: bottom row, iteration number is x multiplied by 1024)

TABLE I. CONFUSION MATRIX BETWEEN SOM AND MLH

\begin{tabular}{|c|c|c|c|c|c|c|}
\hline & \multicolumn{6}{|l|}{ SOM } \\
\hline \multirow{6}{*}{ MLH } & & structure & road & paddy & soil & water \\
\hline & structure & $94 \%$ & $4 \%$ & $0 \%$ & $0 \%$ & $2 \%$ \\
\hline & road & $1 \%$ & $94 \%$ & $5 \%$ & $0 \%$ & $0 \%$ \\
\hline & paddy & $0 \%$ & $8 \%$ & $92 \%$ & $0 \%$ & $0 \%$ \\
\hline & soil & $3 \%$ & $0 \%$ & $0 \%$ & $64 \%$ & $33 \%$ \\
\hline & water & $0 \%$ & $0 \%$ & $0 \%$ & $0 \%$ & $100 \%$ \\
\hline
\end{tabular}

In this case with the real satellite remote sensing imagery data, it takes much long time (more than 20000 times of iteration is needed) as is shown in Fig. 10.

Also, it is found that there are a few local minima until the SOM learning is converged.

Separability is defined as a ratio between intra cluster variance and between cluster variance. It is also found that the mean of separability ${ }^{6}$, between cluster variance of the proposed SOM based image clustering method with density map is around $16 \%$ better than the existing k-mean clustering as is shown in Table 2.

\footnotetext{
${ }^{6}$ http://arxiv.org/abs/1001.1827
} 


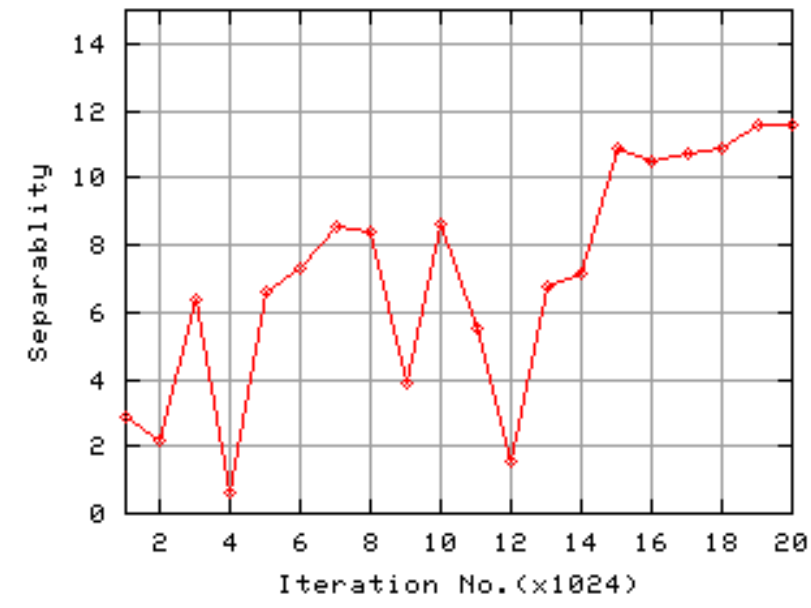

Figure 10 Example of a learning process for Landsat-5 TM imagery data clustering with the proposed SOM based image clustering method.

TABLE II. SEPARABILITY AMONG FIVE CLUSTERS FOR BOTH K-MEAN CLUSTERING AND THE PROPOSED CLUSTERING METHOD FOR LANDSAT TM IMAGERY DATA

\begin{tabular}{|c|c|c|}
\hline & k-mean & SOM \\
\hline Separability & 9.99 & 11.58 \\
\hline
\end{tabular}

\section{CONCLUSION}

A new method for image clustering with density maps derived from Self-Organizing Maps (SOM) is proposed together with a clarification of learning processes during a construction of clusters. It is found that the proposed SOM based image clustering method shows much better clustered result for both simulation and real satellite imagery data. It is also found that the separability among clusters of the proposed method is $16 \%$ longer than the existing $\mathrm{k}$-mean clustering.

It is found that the proposed SOM based image clustering method shows much better clustered result for both simulation and real satellite imagery data. It is also found that the separability among clusters of the proposed method is $16 \%$ longer than the existing k-mean clustering. In accordance with the experimental results with Landsat-5 TM image, it takes more than 20000 of iteration for convergence of the SOM learning processes. Therefore, acceleration of learning process is a next issue for research.

\section{ACKNOWLEDGMENT}

The author would like to thank Dr. Yasunori Terayama for his experimental effort for this research works.

\section{REFERENCES}

[1] Mikio Takagi and Haruhisa Shimoda Edt. Kohei Arai et al., Image Analysis Handbook, The University of Tokyo Publishing Co. Ltd., 1991.,

[2] Kohei Arai, Fundamental Theory for Image Processing Algorithms, Gakujutu-Tosho-Publishing Co. Ltd., 1999.

[3] Kohei Arai, Fundamental Theory for Pattern Recognitions, GakujutuTosho-Publishing Co. Ltd., 1999.

[4] Kohei Arai, Remote Sensing Satellite Image Processing and Analysis, Morikita Publishing Inc., 2001.

[5] T.Kohonen, Self-Organizing Maps, Springer Series in Information Sciences, Vol.30, 1995; Second edition, 1997; Third, extended edition, 2001.

[6] T.Kohonen, G.Barna and R.Chrisley, Statistical pattern recognition with neural networks: benchmarking studies, Proc. ICNN Vol.I, 61-68, 1988.

[7] Kohei Arai, Learning processes of image clustering method with density maps derived from Self-Organizing Mapping(SOM), Journal of Japan Photogrammetry and Remote Sensing, 43, 5, 62-67, 2004.

[8] Kohei Arai, XiangQiang Bu, Pursuit Rainforcement Learning based online clustering for image retrievals, Journal of Image Electronics and Engineering Society of Japan, 39,3,301-309,2010

[9] Kohei Arai, XiangQiang Bu, Pursuit Rainforcement Learning based online clustering with learning automaton for rescue simulations and its accerelation of convergence of learning processes, Journal of Image Electronics and Engineering Society of Japan, 40, 2, 361-168, 2011.

[10] Jouko Lampinen and Timo Kostiainen, Generative probability density model in the Self-Organizing Map. In U. Seiffert and L. Jain, editors, Self-organizing neural networks: Recent advances and applications. pages 75-94. Physica Verlag, 2002.

\section{AUTHORS PROFILE}

Kohei Arai, He received BS, MS and $\mathrm{PhD}$ degrees in 1972, 1974 and 1982, respectively. He was with The Institute for Industrial Science, and Technology of the University of Tokyo from 1974 to 1978 also was with National Space Development Agency of Japan (current JAXA) from 1979 to 1990. During from 1985 to 1987, he was with Canada Centre for Remote Sensing as a Post-Doctoral Fellow of National Science and Engineering Research Council of Canada. He was appointed professor at Department of Information Science, Saga University in 1990. He was appointed councilor for the Aeronautics and Space related to the Technology Committee of the Ministry of Science and Technology during from 1998 to 2000. He was also appointed councilor of Saga University from 2002 and 2003 followed by an executive councilor of the Remote Sensing Society of Japan for 2003 to 2005.

$\mathrm{He}$ is an adjunct professor of University of Arizona, USA since 1998. He also was appointed vice chairman of the Commission "A" of ICSU/COSPAR in 2008. He wrote 30 books and published 332 journal papers. 